\title{
Geometric diagnostics of complex patterns: Spiral defect chaos
}

\author{
Hermann Riecke and Santiago Madruga ${ }^{\text {a) }}$ \\ Engineering Science and Applied Mathematics, Northwestern University, Evanston, Illinois 60208
}

(Received 7 November 2005; accepted 5 January 2006; published online 8 March 2006)

\begin{abstract}
Motivated by the observation of spiral patterns in a wide range of physical, chemical, and biological systems, we present an automated approach that aims at characterizing quantitatively spiral-like elements in complex stripelike patterns. The approach provides the location of the spiral tip and the size of the spiral arms in terms of their arc length and their winding number. In addition, it yields the number of pattern components (Betti number of order 1), as well as their size and certain aspects of their shape. We apply the method to spiral defect chaos in thermally driven RayleighBénard convection and find that the arc length of spirals decreases monotonically with decreasing Prandtl number of the fluid and increasing heating. By contrast, the winding number of the spirals is nonmonotonic in the heating. The distribution function for the number of spirals is significantly narrower than a Poisson distribution. The distribution function for the winding number shows approximately an exponential decay. It depends only weakly on the heating, but strongly on the Prandtl number. Large spirals arise only for larger Prandtl numbers $(\operatorname{Pr} \gtrsim 1)$. In this regime the joint distribution for the spiral length and the winding number exhibits a three-peak structure, indicating the dominance of Archimedean spirals of opposite sign and relatively straight sections. For small Prandtl numbers the distribution function reveals a large number of small compact pattern components. (C) 2006 American Institute of Physics. [DOI: 10.1063/1.2171515]
\end{abstract}

Many systems in nature exhibit complex patterns that may be stationary or time dependent, possibly in a chaotic fashion. To understand these patterns and any transition they might undergo, it is important to have quantitative measures that characterize the relevant properties of the patterns and their time dependence. Due to the multitude of different types of patterns, it is not to be expected that a single measure would be sufficient to capture the qualitatively different aspects of the various patterns. Thus, while quite a few different measures have been developed over the years, so far no convincing approach is available that extracts the characteristic features of patterns dominated by spiral-like pattern components. This paper presents an automated method that allows one to determine quantitatively features like the length of a spiral and its winding number. The approach is, however, not limited to proper spirals and yields additional insightful measures.

\section{INTRODUCTION}

Complex spatio-temporal patterns abound in nature. Many of them also contain chiral elements like spirals or spiral segments. Particularly well-known and beautiful examples are spiral waves in oscillating and excitable chemical systems, ${ }^{1-5}$ chiral structures in microbial growth patterns, ${ }^{6,7}$ and spiral defect chaos in thermally driven convection. 8,9 Other examples are spirals arising in vibrationally excited waves on the surface of granular material, ${ }^{10}$ in calcium waves in oocytes ${ }^{11}$ and hippocampus, ${ }^{12}$ and in the aggrega-

\footnotetext{
a) Present address: Max-Planck-Institute for Physics of Complex Systems, D-01187 Dresden, Germany.
}

tion patterns of the amoeba Dictyostelium. ${ }^{13,14}$ These patterns often evolve chaotically in time and arise in transitions from simpler structures, and may in turn undergo further transitions to other, possibly more complex structures. To gain detailed understanding of such spatio-temporally chaotic states and their transitions, it is important to have quantitative measures that extract the relevant features of the patterns.

Reflecting the variety of spatio-temporal structures observed, a host of different measures and approaches has been developed over the years to characterize the various types of spatio-temporal chaos. Global measures for the disorder in the pattern can be obtained from the correlation function and the spectral entropy. ${ }^{15}$ These measures are quite general; they do not give any local information and do not take any specific features of the patterns into account. For patterns that can be considered as deformed periodic patterns a set of disorder measures has been introduced that is sensitive to the curvature and the deformation of the pattern. ${ }^{16,17}$ In many cases the patterns are locally stripelike. For such patterns approaches have been developed that extract the local wave vector, ${ }^{18,19}$ the orientation of the stripes, ${ }^{20}$ and their curvature. $^{20}$

Many complex patterns exhibit striking, isolated objects like point defects, e.g., dislocations, ${ }^{21,22}$ disclinations, ${ }^{23}$ or penta-hepta defects. ${ }^{24,25}$ Dislocations often take on the form of spirals, as is the case for points of vanishing oscillation amplitude in spatially extended oscillations. ${ }^{26,27}$ Similar spirals also arise in excitable systems. ${ }^{1,28}$ Spiral-defect chaos in convection simultaneously exhibits dislocations, disclinations, and spirals. ${ }^{8}$ In systems displaying such point defects it 
has proven useful to investigate the statistics of the defects or spirals $^{21,22,26,28,29}$ and also of their trajectories. ${ }^{29,30}$

Recently, a different type of characterization has been developed that focuses on the topology of the patterns and extracts the number of contiguous components and of their holes. It has been applied to numerical simulations of spatiotemporal chaos in excitable reaction-diffusion models ${ }^{31}$ and to extensive experiments on spiral defect chaos. ${ }^{32}$

To identify spirals specifically and to measure their characteristics, only a few methods have been used so far. In oscillatory systems in which the spirals emanate from a defect with vanishing oscillation amplitude the defect is relatively easily found and the number of defects can be counted. ${ }^{26}$ In Ref. 33 a fast method is discussed to find the focus of spirals, independent of their dynamical origin, which is based on the evolute of the emitted wave pattern. In principle, spirals can also be detected from singularities in the local orientation of the pattern ${ }^{20}$ or the local wave vector. $^{18}$

In this paper we discuss an approach that allows one to extract typical spiral features like the position of the spiral tip, the size of the spiral measured in terms of the length of the spiral arm, as well as its winding number. The method is, however, not limited to proper spirals, but characterizes arbitrarily shaped segments of stripelike patterns that have a typical wavelength. In the process other measures like the number, size, and compactness of the components of the patterns are obtained as well.

We discuss our diagnostic method at length in Sec. II. In Sec. III we apply it to spiral defect chaos in thermal convection and present the dependence of various measures on the heating and on the Prandtl number of the fluid. We show that the distribution function for the number of spirals in the pattern is narrower than a Poisson distribution. The distribution of the winding number of the spirals is reasonably approximated by an exponential, as had been suggested by experiments. ${ }^{34}$ We find that while the decay rate depends only weakly on the heating it does depend strongly on the Prandtl number. Correlating the contour length and the area of the components we find the signature of target patterns for $\operatorname{Pr}=1.5$, but not for $\operatorname{Pr}=0.3$. For $\operatorname{Pr}=1.5$ the joint distribution for the spiral length and the winding number reveals that Archimedean spirals and relatively straight components dominate the patterns, while for $\operatorname{Pr}=0.3$ no such distinct groups are found. Instead, in this regime the patterns are characterized by a large number of small compact components. In Sec. IV we discuss our conclusions.

\section{METHOD}

Our main goal is to identify spiral-like structures in a sequence of patterns and to characterize them quantitatively. In the process we also obtain some other measures of the patterns. Here, we describe the procedure in detail. It is based on a set of contour lines giving the pattern. Spiral arms are defined via the tip at the point of highest curvature and the merging of the arm with other parts of the pattern at a vertex [cf. Fig. 1(b) below].

We start from a sequence of snapshots that characterizes some continuous quantity of the system. In our example of convection it is the temperature field in the midplane of the convection cell. While in many pattern analyses such a continuous representation is first reduced to a binary representation, we use the continuous representation to obtain the set of contour lines corresponding to the level midway between the minimal and the maximal value of the field taken across all frames of the sequence. The resulting contour lines retain more spatial resolution than the interface of a binary pattern. Our simulations of the convection system employ periodic boundary conditions. To reflect this periodicity, we appropriately connect contours that reach the boundaries. Most contours become closed in this process. In many patterns there are, however, also a few open contours; they wrap around the whole system and may, for instance, correspond to a straight convection roll that spans the system and connects with itself across the periodic boundaries.

Having obtained all closed contours, we already are in the position to obtain a few simple quantitative characterizations of the pattern. The number of closed contours provides a topological measure of the pattern by giving its number of components. In many patterns it is useful to distinguish between "white" and "black" components, e.g., contours inside of which the temperature is higher (lower) than the level of the contour. Focusing, for instance, on the pattern made up of the white components, the number of white components gives then the Betti number of order 1, while in our case of periodic boundary conditions the number of black components is related to the Betti number of order 2, which counts the number of holes in a pattern. ${ }^{31,32}$ The number of black components also includes black components that wrap around the periodic system and close upon themselves. They do not constitute holes of the white pattern and do not count towards the Betti number of order 2. We apply our diagnostics to Boussinesq convection, which has an up-down symmetry that renders up- and downflows equivalent. Therefore, the statistics of white and black components are the same.

It is often useful to go beyond the topological characterization of the patterns and also measure metric aspects like the size of components. We therefore keep track also of the length $\mathcal{P}$ of the perimeter of each component, i.e., the length of the enclosing contour, and of its area $\mathcal{A}$. Comparing the length and area of a given closed contour gives a measure for the compactness of the component, which we define as

$$
\mathcal{C}=4 \pi \frac{\mathcal{A}}{\mathcal{P}^{2}} .
$$

With this normalization a circle has $\mathcal{C}=1$, while small values of $\mathcal{C}$ correspond to filamentary structures.

To extract spirals from the contour lines we start by finding the tips of the spirals, which we identify with the points of highest positive (convex) curvature of the contour. In disordered patterns and, in particular, in noisy patterns, points with high curvature may also appear away from the tips. To reduce the number of these spurious tips we first smooth the contour. To do so we "diffusively" move the polygon points $\left(x_{i}, y_{i}\right), i=1, \ldots N$, which define the contour, using the mapping 


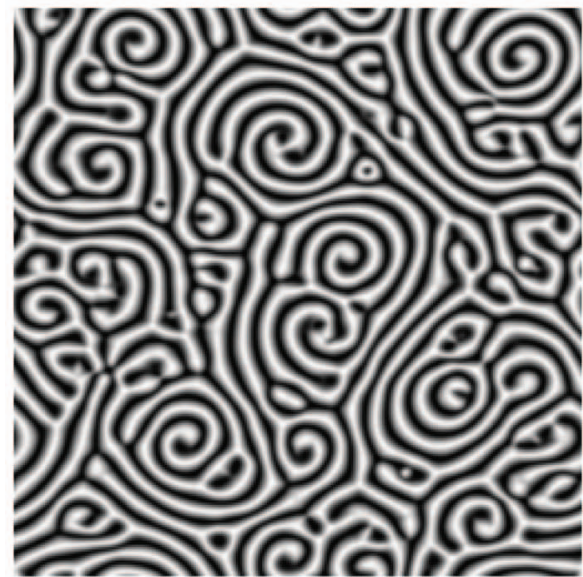

(a)

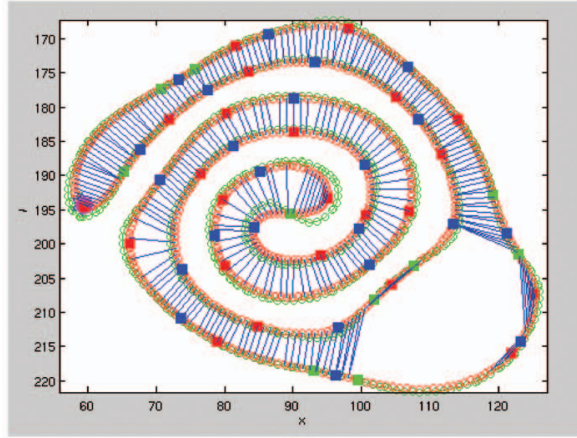

(b)

FIG. 1. (Color) (a) Example of a convection pattern for $\operatorname{Pr}=1.0$ and $\epsilon$ $=1.0$ exhibiting spiral defect chaos. (b) Example of a component in the bottom-left-quadrant of the pattern in (a). Original contour (green open circles), smoothed contour (red open circles), local maxima and minima of the curvature (red and blue solid squares, respectively), inflection points (green solid squares), shortest distance across the components (blue lines). The blue lines also mark the two individual components of this contour and the vertices at which they end [note the black inclusion in (a)] as well as a spurious spiral tip (bottom right). Here, the axes are in terms of the grid coordinates rather than physical coordinates.

$$
x_{i} \rightarrow x_{i}+\delta\left(x_{i+1}-2 x_{i}+x_{i-1}\right),
$$

and analogously for $y_{i}$. To avoid overshoots we use a small value of $\delta$, typically $\delta=0.2$, and perform the smoothing iteratively (typically in 50 steps). While the smoothing strongly reduces localized bulges along overall straight sections of the contour and therefore reduces the number of spurious tips, the curvature at a spiral tip is not affected much. Note that small components shrink substantially by the smoothing. In this procedure we are, however, mostly interested in large components and their reduction in size is quite small. This is illustrated in Fig. 1(b), which depicts a white component in the bottom-left quadrant of the pattern shown of Fig. 1(a) before (green open points) and after (red open circles) the smoothing. A further benefit of the smoothing is that it distributes the grid points $\left(x_{i}, y_{i}\right)$ more equally along the contours.

To obtain the local curvature of the contour, we parametrize for each point $\left(x_{i}, y_{i}\right)$ of the contour a small section of the contour consisting of 5 to 11 points around $\left(x_{i}, y_{i}\right)$ by the arc length $s$ and perform a best fit with two quadratic polynomials $[x(s), y(s)]$. The local curvature is then given by

$$
\kappa_{i}=\frac{x^{\prime} x^{\prime \prime}-y^{\prime} y^{\prime \prime}}{{\sqrt{x^{\prime 2}+y^{\prime 2}}}^{3}},
$$

where the primes denote first and second derivatives of $x(s)$ and $y(s)$, respectively, at the midpoint $\left(x\left(s_{m}\right), y\left(s_{m}\right)\right)$ of the arc approximating the contour over this local range.

We then identify the spiral tips contained in this contour iteratively by locating the point with maximal positive curvature. To measure the length of this spiral arm we follow both branches of the contour originating from the tip until the first vertex is reached [cf. Fig. 1(b)]. Vertices are identified by the width of the arm exceeding a threshold $w_{\max }$. Typically we take a value of $w_{\max }=10$, which corresponds to about one wavelength of the pattern. To measure the width we find for each point on one branch emanating from the spiral tip the closest point on the other. In general this is a nonlocal problem. It becomes much simpler by starting from the tip $\left(x_{i_{i t p}}, y_{i_{\text {tip }}}\right)$ and finding for each consecutive point $P_{j}^{(l)}$ $\equiv\left(x_{j}^{(l)}, y_{j}^{(l)}\right), j<i_{\text {tip }}$, on the "lower" branch the point $P_{k}^{(u)}\left(P_{j}^{(l)}\right)$ on the "upper" branch that is closest to $P_{j}^{(l)}$ and that is within a certain range $i_{\text {tip }}<k^{\prime} \leqslant k \leqslant k^{\prime}+\Delta k$ of the previously identified point $P_{k^{\prime}}^{(u)}\left(P_{j-1}^{(l)}\right)$ on the upper branch. We typically take $\Delta k=5$. In Fig. 1(b) the closest points identified in this manner are connected by blue lines. Near the tip this procedure sometimes leads to connections that lie outside rather than inside the contour. To aid avoiding this problem, we require that within a core region near the tip the point on the upper branch strictly advance, $k>k^{\prime}$. Typically we take the core region to be 10 points. When reaching a vertex, typically the points on the lower branch still advance while those identified as closest on the upper branch do not [e.g., near $x=115 y=197$ in Fig. 1(b)]. We then identify the end of the spiral arm with the last pair of points $\left[P_{j}^{(l)}, P_{k}^{(u)}\left(P_{j}^{(l)}\right)\right]$ for which the point on the upper branch still advanced, $P_{k}^{(u)}\left(P_{j}^{(l)}\right) \neq P_{k^{\prime}}^{(u)}\left(P_{j-1}^{(l)}\right)$. For a segment or spiral arm thus identified, we take its length $\mathcal{S}$ to be the mean of the arc length along its two branches and define its winding number via $\mathcal{W} \equiv \frac{1}{2}\left(\theta^{(l)}+\theta^{(u)}\right) / 2 \pi$, where $\theta^{(l)}$ and $\theta^{(u)}$ are the angles by which the two branches of the contour are rotated from the tip to the vertex, respectively.

Having found the first tip and the associated spiral arm, we then identify the second spiral tip with the point of maximal positive curvature among the points on the contour not included in this first arm and correspondingly for further arms. When identifying the subsequent spiral arms we ensure that they do not overlap with previously identified arms. In many contours there are portions that are not associated with any spiral tip. Nevertheless, even in these parts of the contour the algorithm may identify spiral segments, i.e., segments that are connected with local positive maxima of the curvature, which are, however, spurious. Figure 1(b) shows such a segment in the lower right (marked by the blue lines indicating the shortest distance across the component). It is associated with a black inclusion. The size of the segments generated in this way is of the order of $w_{\max }$. To exclude such segments, we omit all segments with an arc length smaller than $2 w_{\max }$ in the final analysis of the results. 
Many contours do not include a vertex. In those cases we associate the contour with two segments (spiral arms) by cutting the contour at the point $P_{j}^{(l)}\left[\right.$ and $\left.P_{k}^{(u)}\left(P_{j}^{(l)}\right)\right]$ at which the absolute value of the curvature outside the core regions of the two tips is minimal.

The spirals of spiral defect chaos in convection are not topologically stable objects; their winding number varies continuously and is not conserved. In other systems spirals are topologically stable and can only be created in pairs with opposite topological charge. Well-studied systems of this type are oscillations in extended media in which the spiral cores represent singularities of the phase of oscillation at which the oscillation magnitude vanishes. ${ }^{26}$ In particular in such cases it is of interest to count the number of spirals with positive and negative charge independent of their size. Employing the algorithm discussed here, the topological charge of the spirals can be related to the location of inflection points of the contour in the vicinity of the spiral tip. Even in quite small spirals the curvature changes sign near the tip, and the side on which this inflection point occurs depends on the topological charge of the spiral. Note, however, that even segments that have a noticeable winding number may not be classified as spirals according to this criterion if their tip is part of a relatively straight section. An example of such a segment is seen in Fig. 1(b), where the segment with winding number $\mathcal{W} \sim 0.25$ is quite straight near its tip, and consequently the first inflection point (marked with a green solid circle) is relatively far from the tip.

\section{GEOMETRIC ANALYSIS OF SPIRAL DEFECT CHAOS}

We use the approach discussed in Sec. II to analyze spiral defect chaos in Boussinesq convection, ${ }^{8,9}$ which is characterized by two system parameters: the Prandtl number Pr of the fluid, which measures the ratio between its viscosity and its heat conductivity, and the heating, which is measured by the dimensionless Rayleigh number $R$. The latter is often expressed in terms of the scaled distance $\epsilon$ from the onset of convection at $R_{c}=1708, \epsilon \equiv\left(R-R_{c}\right) / R_{c}$.

Our results are based on direct numerical simulations of the Navier-Stokes equations employing a pseudospectral code developed by Pesch and co-workers. ${ }^{35}$ Details of the equations and the code can be found in Refs. 36 and 37. The code is based on Fourier modes in the horizontal direction and appropriate combinations of trigonometric and Chandrasekhar functions that satisfy the top and bottom boundary conditions in the vertical directions. ${ }^{38,39}$ All runs are performed with $256 \times 256$ horizontal Fourier modes for a system size of $L=32 \cdot 2 \pi / q_{c}$, where $q_{c}=3.098$ is the critical wave number, and two vertical modes. Previous simulations have shown that this spatial resolution is sufficient to capture the spiral defect chaos at the very least semiquantitatively. ${ }^{35,36}$ Since the data analysis requires relatively long runs, going to a significantly higher spatial resolution is beyond our current computational means. To solve for the time dependence the code uses a fully implicit scheme for the linear terms, whereas the nonlinear parts are treated explicitly using a second-order Adams-Bashforth method. The time step is typically taken to be $t_{v} / 500$, where

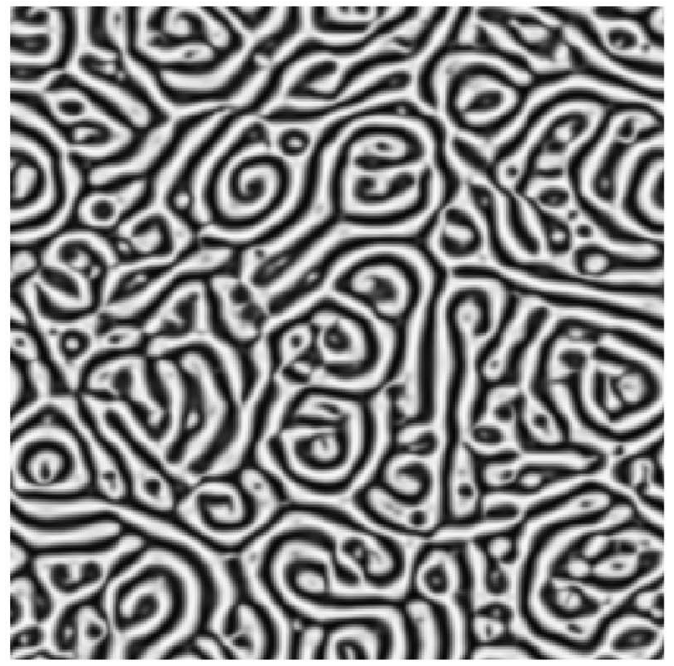

(a)

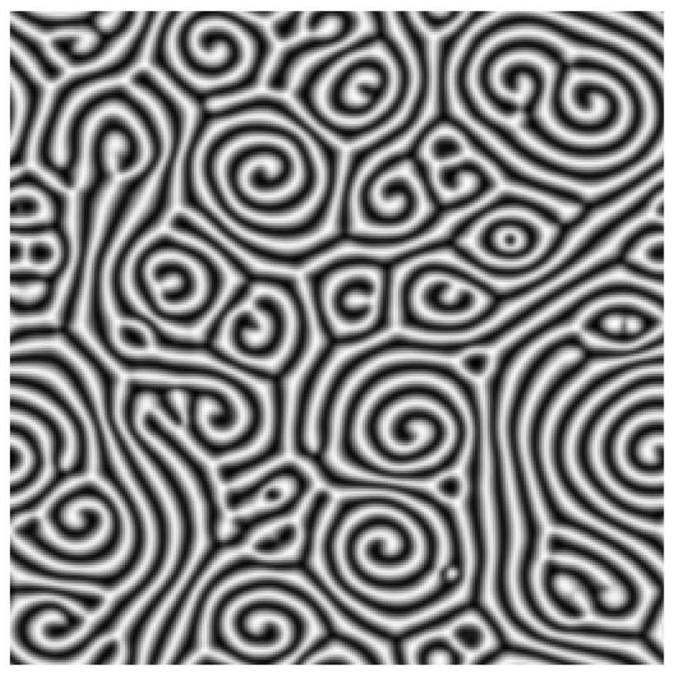

(b)

FIG. 2. Snapshots of convection patterns at $\epsilon=1$ for (a) $\operatorname{Pr}=0.3$ and (b) $\operatorname{Pr}$ $=1.5$. System size $L=64.9$.

$t_{v}$ is the vertical diffusion time. In our analysis we use snapshots of the temperature field in the midplane of the convection cell that are typically taken every $2 t_{v}$. We focus on three different values of $\epsilon$ and $\operatorname{Pr}$ each. The number of snapshots evaluated range from 500 for $\operatorname{Pr}=0.3$ to 2000 for $\operatorname{Pr}=1.5$. Examples of patterns at $\epsilon=1.0$ are shown in Fig. 1(a) for $\operatorname{Pr}=1.0$ and in Figs. 2(a) and 2(b) for $\operatorname{Pr}=0.3$ and $\operatorname{Pr}=1.5$.

We first discuss the results for various mean quantities. Figure 3 shows the dependence of the mean number $\mathcal{N}$ of closed contours on $\epsilon$ for three different Prandtl numbers. More precisely, $\mathcal{N}$ gives the mean $\left(\mathcal{N}_{b}+\mathcal{N}_{w}\right) / 2$ of "black" and "white" components. To assess the variations in $\mathcal{N}$ over the course of the simulation, Fig. 3 shows in small symbols the mean values obtained from averaging only over the initial, middle, and final third of the run. In most cases the variations are so small that the additional symbols are barely visible. The number of closed contours increases with increasing $\epsilon$ and decreasing Pr. This is consistent with the fact that the driving force of spiral defect chaos is a large-scale flow, which is driven by the curvature of the convection rolls 


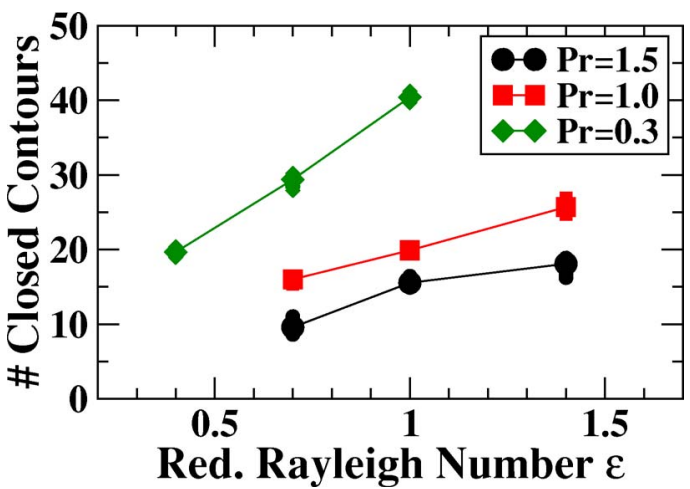

FIG. 3. (Color online) Mean number of closed contours as a function of $\epsilon$ for different values of the Prandtl number Pr.

and which increases with increasing $\epsilon$ and decreasing Pr. Such an $\epsilon$ dependence has also been found in recent experiments for $\operatorname{Pr} \sim 1 .^{32}$ There, the Betti numbers of order 1 and 2 have been measured, which give the number of contiguous components and of holes in the pattern, respectively. ${ }^{31}$ The mean length of the contours exhibits the opposite behavior (data not shown). In Fig. 4 the analogous trend of increasingly finer structures with increasing strength of the largescale flow is depicted in terms of the mean arc length of the segments (spirals) of the components.

The arc length results for $\operatorname{Pr}=1.5$ show a large scatter at $\epsilon=0.7$. This reflects the fact that for this system size $\epsilon=0.7$ is close to the onset of spiral defect chaos. ${ }^{40}$ Figure 5 shows that in this regime the pattern does not exhibit much of a spiral character. Our computational resources do not allow us to establish whether this low chaotic activity persists indefinitely. Alternatively, the pattern could eventually freeze in a disordered pattern. Experimentally, it has been found that near the transition to spiral defect chaos the system can exhibit very intermittent behavior in which long periods of almost-ordered patterns alternate with states exhibiting a few spirals. $^{40}$

To extract the spiral character of the chaotic patterns we measure their winding number. Since the system is chirally symmetric the mean winding number is very close to 0 in all cases. The spiral character is therefore better captured by the standard deviation of the winding number. Its dependence on $\epsilon$ and $\operatorname{Pr}$ is given in Fig. 6. Two trends can be discerned. For the strongly chaotic states, i.e., for low Prandtl number and

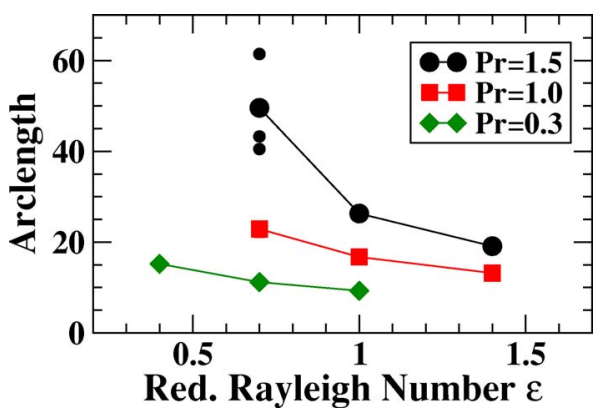

FIG. 4. (Color online) Mean arc length $\mathcal{S}$ of the segments of the components as a function of $\epsilon$ for different values of the Prandtl number Pr.

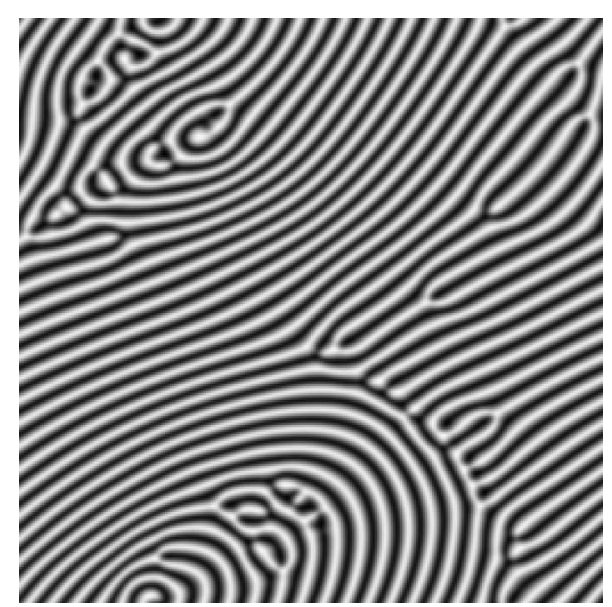

FIG. 5. Snapshot of a typical convection pattern for $\operatorname{Pr}=1.5$ and $\epsilon=0.7$.

large $\epsilon$, the standard deviation of the winding number decreases with increasing $\epsilon$ and decreasing Pr, similar to the dependence of the arc length. This reflects the fact that shorter spiral segments have a smaller winding number. For low chaotic activity, however, the winding number decreases with decreasing $\epsilon$, even though the arc length continues to increase with decreasing $\epsilon$. This is very striking for $\operatorname{Pr}=1.5$ and $\epsilon=0.7$ and consistent with the visual appearance of the pattern shown in Fig. 5. But, even for $\operatorname{Pr}=1.0$ the spiral character of the state is nonmonotonic in $\epsilon$. For these parameter values no tendency for the pattern to become ordered was apparent visually. Thus, the standard deviation of the winding number may be suitable as a sensitive quantitative measure for the transition to spiral defect chaos, complementing the previously used mean number of spirals. ${ }^{40}$

A natural conjecture is that the dependence of the various measures on $\epsilon$ and $\operatorname{Pr}$ would be simpler if they were considered as functions of the distance from the onset of spiral defect chaos, $\epsilon-\epsilon_{\mathrm{SDC}}(\mathrm{Pr})$. To test this in detail would require a quantitative measurement of $\epsilon_{\mathrm{SDC}}(\mathrm{Pr})$. This is beyond our computational capabilities. However, while both the mean number of components and the arc length take on comparable values for $(\operatorname{Pr}=0.3, \epsilon=0.4),(\operatorname{Pr}=1.0, \epsilon=1.0)$, and $(\operatorname{Pr}=1.5, \epsilon=1.4)$, this is not the case for the standard deviation of the winding number, which reaches significantly higher overall values for $\operatorname{Pr}=1.5$ than for $\operatorname{Pr}=0.3$. Thus, the values at different Prandtl numbers cannot be related to each

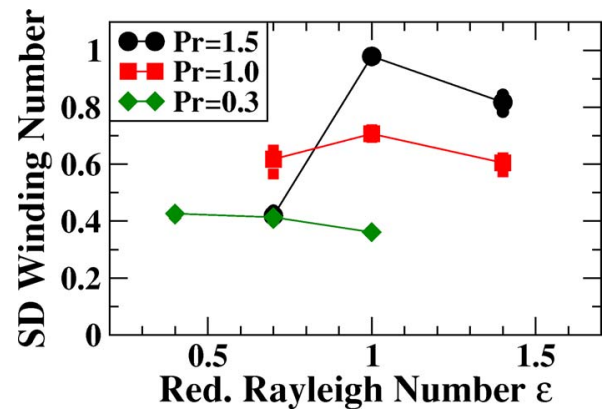

FIG. 6. (Color online) Standard deviation of the winding number as a function of $\epsilon$ for different values of the Prandtl number Pr. 


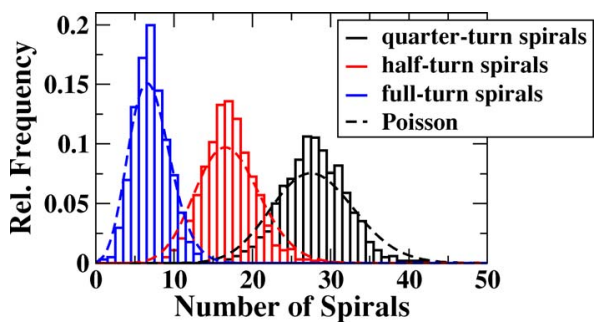

FIG. 7. (Color online) Distribution function for the number of spirals for three different values of the threshold $\mathcal{W}_{\text {min }}$ for $\operatorname{Pr}=1.0$ and $\epsilon=1.0$. Dashed lines give fits to a Poisson distribution.

other by a simple Pr-dependent shift and rescaling of $\epsilon$, indicating a somewhat more complex scenario (see also Figs. 12 and 13 below).

In wave systems the magnitude of the complex amplitude of the oscillations vanishes at the spiral core. The spirals then constitute topologically conserved defects and are equivalent to dislocations of stripe patterns. For the characterization of spatio-temporal chaos in systems with such topological defects, it has proven quite instructive to measure the distribution function for the number of spirals or dislocations, respectively, in a given snapshot. ${ }^{21,22,26,28,29}$ In various, but not all ${ }^{28,29}$ systems the distribution function was found to be well fit by a squared Poisson distribution, which is consistent with a simple overall behavior of the spirals: they are created pairwise with a fixed probability, diffuse randomly, and annihilate each other pairwise upon collisions. The spirals in spiral defect chaos are not topologically conserved and may exhibit different statistics. Indeed, in a substantial effort the distribution function was measured previously by visual inspection of many experimentally obtained snapshots. ${ }^{41}$ There, it was found for $\operatorname{Pr}=0.96$ in a system with aspect ratio $\Gamma=52$ that the probability distribution function was consistent with a Poisson distribution for smaller values of $\epsilon(\epsilon=0.72$ and $\epsilon=0.80)$, while for $\epsilon=0.96$ the distribution was more sharply peaked than a Poisson distribution. Of necessity, the manual counting limited the number of snapshots that could be analyzed and the results have a somewhat larger margin of uncertainty. In addition, the distribution was determined only for spiral arms with a minimal winding number of $\mathcal{W}_{\min }=1$. To address the question of the distribution function more precisely and its dependence on the selection criterion for the spirals, we show in Fig. 7 the distribution function for three different values of the thresh-

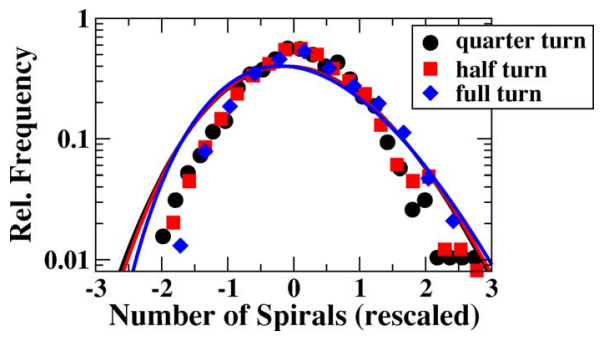

FIG. 8. (Color online) Rescaled distribution function for the number of spirals for the three different values for the threshold $\mathcal{W}_{\min }$. The points and curves are obtained from those of Fig. 7 by shifting and rescaling by the mean of the distribution.
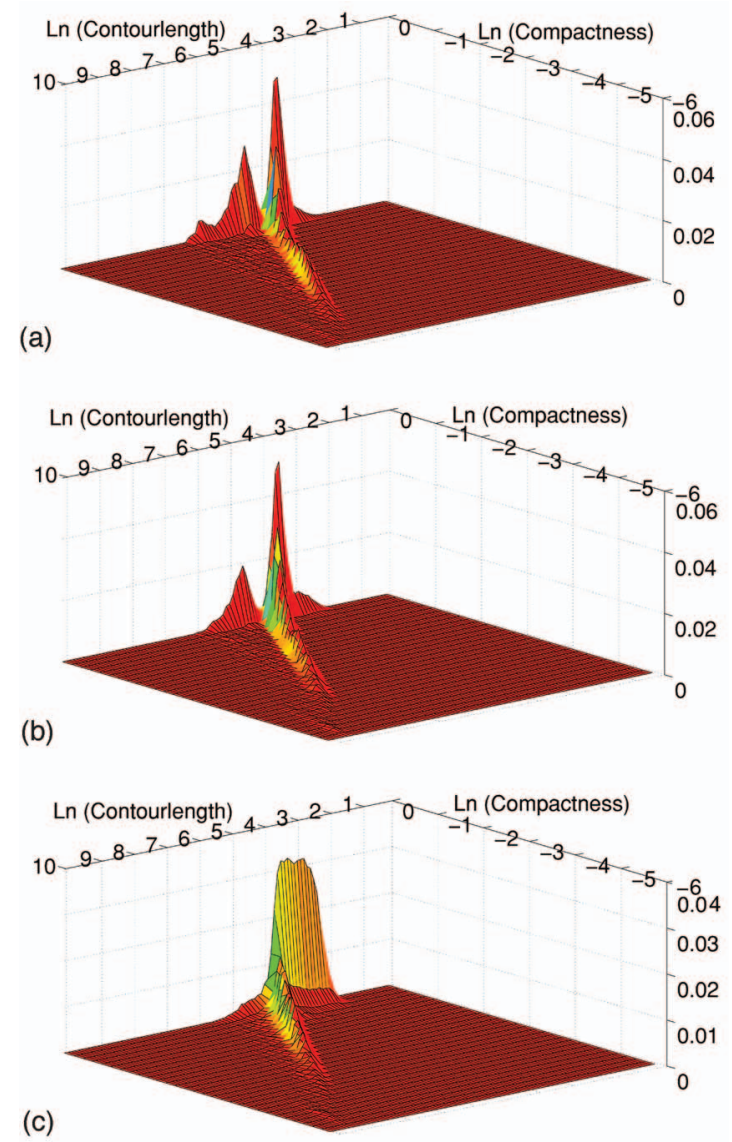

FIG. 9. (Color) Joint distribution function for the logarithm of the compactness, $\ln \mathcal{C}$, and the logarithm of the contour length, $\ln \mathcal{P}$, for $\epsilon=1.0$. (a) $\operatorname{Pr}$ $=1.5$; (b) $\operatorname{Pr}=1.0$; (c) $\operatorname{Pr}=0.3$.

old, $\mathcal{W}_{\min }=\frac{1}{4}, \mathcal{W}_{\min }=\frac{1}{2}$, and $\mathcal{W}_{\min }=1(\operatorname{Pr}=1.0$ and $\epsilon=1.0)$. Our results confirm with higher accuracy the experimental finding that the distribution function is narrower than a Poisson distribution. Moreover, shifting the distribution functions by their means and rescaling them by the square root of their means, which would correspond to the standard deviation for a Poisson distribution, results in the distributions shown in Fig. 8 (note the logarithmic scaling). Within the statistical error the distribution functions collapse independent of the threshold $\mathcal{W}_{\min }$ and are significantly more sharply peaked than the Poisson distribution. So far no convincing model

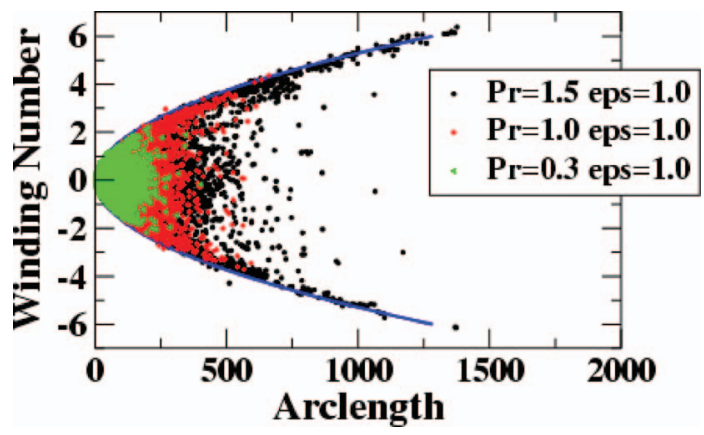

FIG. 10. (Color) Correlation between arc length and winding number for $\epsilon=1.0$. Each dot represents one segment of a component. The line is a fit to an Archimedean spiral [cf. (5)]. 


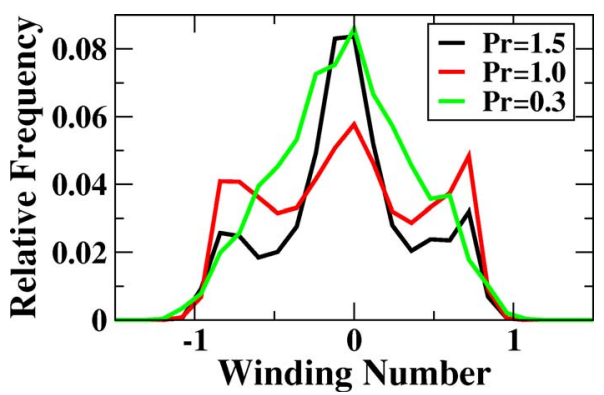

FIG. 11. (Color online) Distribution function of the winding number for arc lengths $\mathcal{S}$ in the range $10 \leqslant \mathcal{S} \leqslant 12.5$ (cf. Fig. 10).

has been put forward that provides an understanding of the form of these distribution functions. Note that for the mean number of defects found in our simulations the difference between the squared Poisson distribution and the usual Poisson distribution is very small and cannot account for the deviations of the measured distribution function from the Poisson fit.

Further insight into the patterns and their dependence on the system parameters can be gained by considering correlations between various properties. An instructive example is the joint distribution function for the logarithm of the compactness of the closed contours and the logarithm of their contour length, which is shown in Fig. 9. We find that most components have a compactness $\mathcal{C} \sim 1$ and small contour length. For larger contour lengths a relatively straight ridge is found in the distribution function. It reflects the fact that the stripes have a typical width $\lambda / 2$. Thus, for large values of $\mathcal{P}$ the compactness $\mathcal{C}$ can be approximated by

$$
\mathcal{C} \sim 4 \pi \frac{\lambda \mathcal{P}}{4 \mathcal{P}^{2}} \propto \mathcal{P}^{-1} .
$$

A few large components deviate from this relationship and have a larger compactness; no component was found that has a smaller compactness. This asymmetry arises because the wavelength of the pattern cannot become smaller than a certain minimal value. For large Prandtl number an additional structure is apparent: for values of the compactness $\mathcal{C} \lesssim 1$ the distribution function shows three quite distinct peaks in its dependence on the contour length. We associate these peaks with the appearance of target patterns, which are comprised of a discrete set of quite compact contour lines. When the Prandtl number is decreased these peaks become less pro-

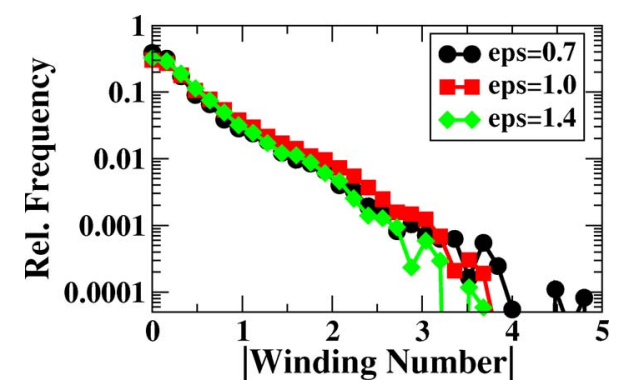

FIG. 12. (Color online) Distribution function of the winding number for $\operatorname{Pr}=1.0$ and different values of $\epsilon$

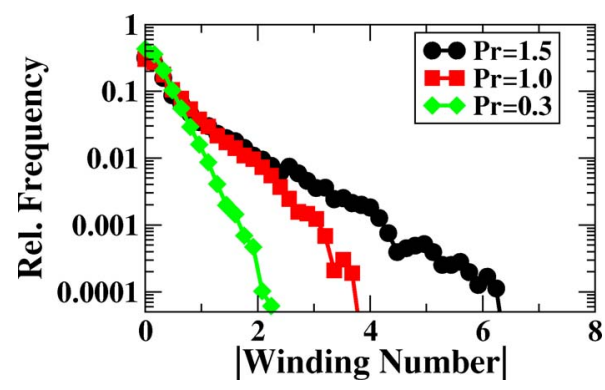

FIG. 13. (Color online) Distribution function of the winding number for $\epsilon$ $=1.0$ and different values of Pr.

nounced [cf. $\operatorname{Pr}=1.0$ in Fig. 9(b)] and disappear for $\operatorname{Pr}=0.3$ [Fig. 9(c)]. Instead, the main peak becomes quite broad and extends towards small contour lengths, indicating the appearance of relatively small compact components. Comparing the distribution functions for different $\mathrm{Pr}$ it is also apparent that the relative frequency of large components decreases noticeably with decreasing Prandtl number.

Direct information about the spiral character of the patterns is obtained by considering the correlation between the winding number and the arc length of the segments. This is shown in Fig. 10 for three Prandtl numbers at $\epsilon=1.0$. Since the wavelength of the stripes is essentially fixed, the winding number $\mathcal{W}$ of any spiral is expected to be limited by that of an Archimedean spiral with the same arc length $\mathcal{S}$. A simple computation gives

$$
\begin{aligned}
\mathcal{S}= & \pi \mathcal{W} \alpha \sqrt{4 \pi^{2} \mathcal{W}^{2}+1}-\frac{1}{2} \alpha \ln \alpha+\frac{1}{2} \alpha \ln (2 \pi \alpha \mathcal{W} \\
& \left.+\alpha \sqrt{4 \pi^{2} \mathcal{W}^{2}+1}\right) .
\end{aligned}
$$

As Fig. 10 shows, Eq. (5) fits the extremal values of $\mathcal{W}$ quite well for all three Prandtl numbers. The fitted value of $\alpha$ $=1.8$ corresponds to a wave number $q=2.2$ for the rolls making up the spiral, which is consistent with the peak of the azimuthally averaged wave vector spectrum measured experimentally. ${ }^{9}$ A cut through the distribution function covering the range $10 \leqslant \mathcal{S} \leqslant 12.5$ is shown in Fig. 11 for three values of $\operatorname{Pr}$. For $\operatorname{Pr}=1.5$ it has three peaks. The outer two correspond to near-Archimedean spirals while the center one to quite straight structures. As the Prandtl number is decreased the triple-peak structure disappears and no signature of the Archimedean spiral is left. As the arc length $\mathcal{S}$ is

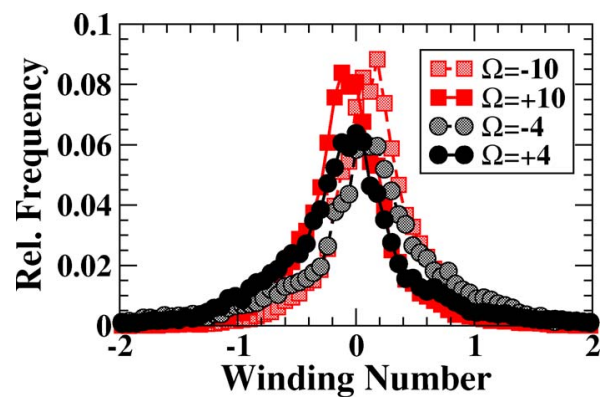

FIG. 14. (Color online) Breaking the chiral symmetry. Distribution function of the winding number for different rotation rates of the system $(\operatorname{Pr}=1.0$ and $\epsilon=1.0)$. 


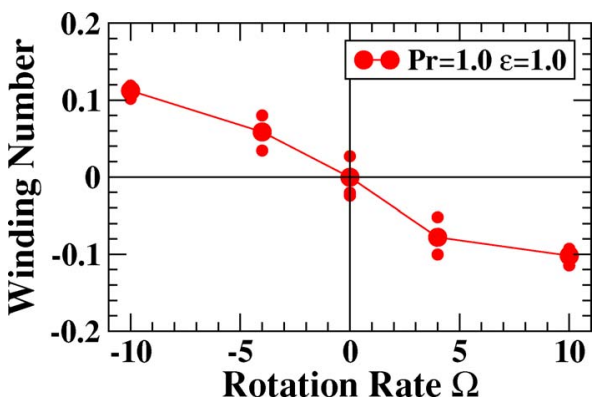

FIG. 15. (Color online) Dependence of the mean winding number on the rotation rate for $\operatorname{Pr}=1.0$ and $\epsilon=1.0$. Small symbols indicate the means over the initial, middle, and final part of the run, respectively.

increased the distribution function drops off very rapidly for $\operatorname{Pr}=0.3$ and, as mentioned before, spirals with winding numbers well above 1 are found only for large Prandtl numbers.

In experiments using $\mathrm{CO}_{2}$ with $\mathrm{Pr}=0.98$ it has been found that for $\epsilon=0.72$ and $\epsilon=0.84$ the distribution function for the winding number decays approximately exponentially. ${ }^{41}$ Note that in Fig. 10 of Ref. 41 the variable $m$ denotes phase windings of $\pi$ and not of $2 \pi$. Due to the manual counting of the spirals only a limited amount of data was available. We have extracted this distribution function also from our data. In Fig. 12 we show the distribution for the magnitude of the winding number for three values of $\epsilon$ for $\operatorname{Pr}=1.0$. In particular, for $\epsilon=0.7$ quite convincing exponential behavior is found. With increasing $\epsilon$ the function decays, however, somewhat faster at large winding numbers, casting some doubt on the exponential behavior. Overall, there is, however, little change with $\epsilon$ and fits to exponentials would lead to similar decay constants. By contrast, when considering different values of the Prandtl numbers quite different results are obtained as shown in Fig. 13, where we keep $\epsilon$ fixed at $\epsilon=1.0$. With increasing Prandtl number the decay of the distribution function becomes significantly slower and spirals with larger winding numbers become much more likely, as might have been surmised from Fig. 10. Thus, as became apparent already in the discussion of Figs. 3,4 , and 6 , the state of spiral defect chaos depends on the Prandtl number and $\epsilon$ separately and not only on the distance $\epsilon-\epsilon_{\mathrm{SDC}}(\mathrm{Pr})$ from its onset.

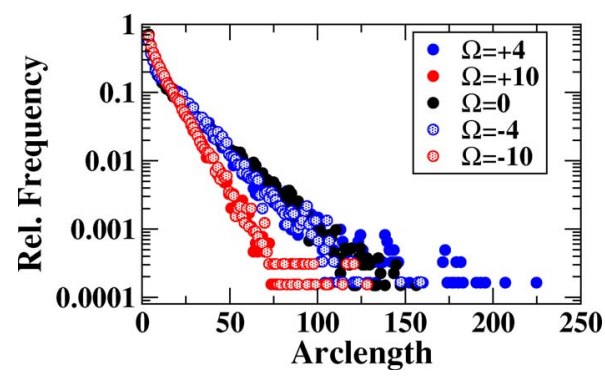

FIG. 16. (Color online) Distribution function of the arc length of the spiral segments for different rotation rates $(\operatorname{Pr}=1.0$ and $\epsilon=1.0)$.

Since the spirals break the chiral symmetry a particularly interesting question is how they respond to an external breaking of the chiral symmetry as it arises in a rotating convection system. This question has been addressed previously in experiments using $\mathrm{CO}_{2}$ at $\mathrm{Pr}=0.98$. There the number of clockwise and counterclockwise spirals was counted by hand and it was found that the rotation introduces an imbalance between the two populations. ${ }^{42}$ In terms of the winding number we expect a smooth shift of the distribution function with increasing rotation rate $\Omega$, as is indeed borne out in Fig. 14. The resulting dependence of the mean winding number on the rotation rate is shown in Fig. 15.

As shown in Fig. 15, with increasing $|\Omega|$ the mean winding number decreases sublinearly. This is connected with the fact that the segments of the pattern become shorter with increasing rotation rate in anticipation of the Küppers-Lortz instability. ${ }^{43}$ This decrease is shown in Fig. 16, which presents the distribution function for the arc length for different rotation rates. Note that for $\operatorname{Pr} \sim 1$ the onset of the KüppersLortz instability is not reached until $\Omega \sim 14 .{ }^{9}$ If instead of the mean winding number the mean topological charge of the patterns is plotted an essentially linear behavior is found (data not shown).

So far we have focused in our analysis on the temperature field in the midplane $z=0$ of the convective layer. While it seems reasonable to assume that the pattern in the midplane is representative for the convective patterns, we test this assumption explicitly by measuring the mean number of closed contours (white components, black components, and the mean of the two), the mean number of spirals with $\mathcal{W}$

TABLE I. Dependence of the mean number of closed contours [positive, negative, and average of the two (cf. Fig. 3)], mean number of spirals, the arc length, and the standard deviation of the winding number on the vertical position of the temperature field. The first four columns of the table give the ratio between the quantity at the indicated $z$ level and at the midplane $(z=0)$. The fifth column gives the corresponding ratios for the temperature field averaged over the five $z$ levels. The last column gives the value at the midplane ( $\mathrm{Pr}$ $=0.80, \epsilon=1.4)$.

\begin{tabular}{lllllll}
\hline \hline & $z=-0.25$ & $z=0.25$ & $z=-0.125$ & $z=0.125$ & $\langle z\rangle$ & $z=0$ \\
\hline \# Contours (white) & 1.40 & 0.733 & 1.14 & 0.876 & 1.00 & 7.15 \\
\# Contours (black) & 0.738 & 1.32 & 0.896 & 1.13 & 1.008 & 7.28 \\
\# Contours & 1.065 & 1.031 & 1.017 & 1.002 & 1.005 & 7.21 \\
\# Spirals & 1.09 & 1.11 & 1.02 & 1.06 & 1.03 & 1.22 \\
Arc length & 0.996 & 1.03 & 0.993 & 1.02 & 0.993 & 12.21 \\
SD winding number & 1.01 & 1.05 & 1.001 & 1.02 & 0.999 & 0.512 \\
\hline \hline
\end{tabular}


$\geqslant 1$, the mean value of the arc length, and the standard deviation of the winding number also at different heights $z$. For these quantities Table I gives the ratio between the value at the indicated $z$ value and the value at the midplane $z=0$, which is given in the last column. In experiments typically one does not have access to patterns at a certain $z$ level. Instead, the measured patterns correspond to an average across the layer. We therefore also analyzed the patterns obtained by averaging the temperature fields at the five locations listed in the table. The results of those diagnostics are shown in the fifth column, again normalized by the corresponding values at $z=0$. In these runs we used a reduced system size of $L=16 \cdot 2 \pi / q_{c}$ retaining $128 \times 128$ Fourier modes. The Prandtl number is $\operatorname{Pr}=0.8$.

Considering the number of contours enclosing white components, one notices a strong dependence on the height $z$ at which the temperature field is measured. For instance, while at $z=-0.25$ the mean number of white components is $40 \%$ higher than at $z=0$, it is almost $30 \%$ lower at $z=$ +0.25 . However, if one averages over the white and the black components this variation is reduced to $6.5 \%$ or less. The $z$ dependence of the other quantities is similarly low. Since the experimental visualization techniques measure some vertical average of the temperature field, one can expect that the difference between experimentally determined quantities and the computationally obtained ones remains below $10 \%$. For some quantities like the average number of closed contours the variation with $\operatorname{Pr}$ and $\epsilon$ over the range considered is noticeably larger than any of these differences. On the other hand, some quantities like the standard deviation of the winding number vary with $\epsilon$ and Pr only by $10 \%$ or $20 \%$. For these quantities the direct comparison between the computation and experimentally determined values may be not quite as conclusive. However, even if different experimental or computational measurement protocols should lead to results that differ quantitatively from each other, we expect that even less marked trends like the nonmonotonicity of the standard deviation of the winding number (cf. Fig. 6) should be readily resolvable as long as the protocol is kept the same when changing $\epsilon$ or Pr. Moreover, if the average over the five $z$ levels, which is shown in the fifth column of Table I, constitutes a good approximation to the experimentally obtained patterns, the diagnostics of the pattern at $z=0$ should provide a very good prediction of the experimental results.

As a further test of the significance of the $z$ dependence Fig. 17 shows the distribution function for the winding number at $z=0, z=-0.125$, and $z=-0.25$. Within the statistical error these distributions are indistinguishable. We conclude therefore that the measures discussed in this paper will be meaningful and will provide valuable insight into these complex spiral patterns even though the patterns themselvesand with them the measures-vary somewhat across the layer.

\section{CONCLUSION}

Motivated by the fascinating convection patterns found in spiral defect chaos, we have introduced an approach to characterize such patterns based on various features of the

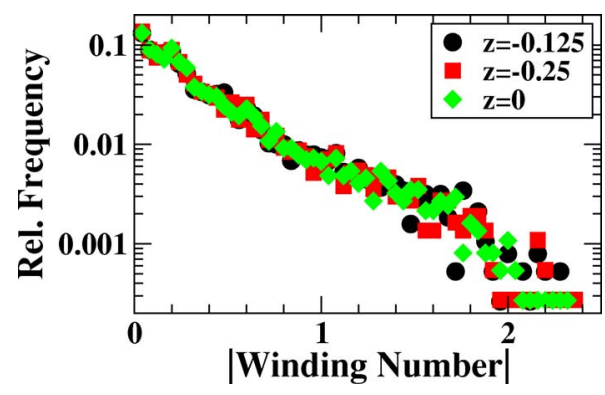

FIG. 17. (Color online) Dependence of the distribution function of the absolute value of the winding number on the vertical position $z$ at which the temperature field is measured $(\operatorname{Pr}=0.8$ and $\epsilon=1.4)$.

spirals. In the process we have introduced other measures that are not specific to spiral-like structures. We have used these various measures to characterize spiral defect chaos as a function of the heating $\epsilon$ and the Prandtl number Pr.

Since the onset $\epsilon_{\mathrm{SDC}}$ of spiral defect chaos depends on the Prandtl number one could surmise that the properties of the resulting spiral defect chaos state depend mostly on the distance $\epsilon-\epsilon_{\mathrm{SDC}}(\operatorname{Pr})$ from that threshold. Comparing the $\epsilon$ dependence of the mean number $\mathcal{N}$ of components of the pattern and of the arc length $\mathcal{S}$ of its segments with the $\epsilon$ dependence of the standard deviation of the winding number of the spirals, we found evidence that this simple relationship is, however, not satisfied. If any scaling relationship should hold at all, it would have to involve also rescaling of the dependent variables. Further support for this conclusion comes from our finding that the distribution function of the winding number is barely affected by changes in $\epsilon$, but it depends significantly on the Prandtl number.

We find that while the arc length of pattern segments increases monotonically as the onset of spiral defect chaos is approached, this is not the case for the winding number of those segments. Thus, further away from threshold the winding number increases with the arc length as $\epsilon$ is decreased, indicating a lengthening of spirals without much change in their structure. However, as the threshold is approached the segments still become longer but their winding number starts to decrease, i.e., the segments become straighter. Possibly, the standard deviation of the winding number could therefore serve as an early indicator for the transition to the stationary state.

With improved accuracy we confirm that the distribution function for the number of spirals is narrower than a Poisson distribution. ${ }^{44}$ Moreover, using different thresholds for the classification of the spirals, we find that after rescaling these different distribution functions collapse into a single curve. So far, no theoretical explanation for this distribution function is available.

Our automated analysis also allows higher accuracy for the measurement of the distribution function of the winding number. In previous experiments a roughly exponential behavior had been found. ${ }^{41}$ Overall we confirm this behavior, but at large winding numbers the distribution functions typically decay somewhat more rapidly. Interestingly, as mentioned above, the decay depends very strongly on the Prandtl number, but very little on $\epsilon$. 
By correlating different measures we presented a more detailed characterization of the patterns. For instance, correlating the contour length of a component and its compactness, which is related to its area, it is quite evident that for large Prandtl numbers the patterns have many filamentary components and a sizable number of target patterns. For small Prandtl numbers the signature of the long filaments and of the target patterns disappears and instead a much broader spectrum of smaller, more compact components arises. In a separate investigation ${ }^{45}$ we find that the number of small compact components strongly increases when the fluid properties depend significantly on the temperature, i.e., when non-Boussinesq effects become important. They introduce a resonant triad interaction between the stripe modes that enhances the tendency towards hexagonal (cellular) patterns. Of course, the breaking of the up-down symmetry by the non-Boussinesq effects leads also to differences between closed contours that enclose upflow rather than downflow regions (black and white components).

Correlating the arc length with the winding number allows the extraction of the spirals. For large Prandtl numbers not only the very long components but also medium-sized components tend to be close to Archimedean spirals. For small Prandtl numbers, however, even among the longer components most have winding numbers that are much smaller than the winding numbers of Archimedean spirals with the same arc length.

A strength of the measures employed in this paper is that they go beyond purely local properties of the contour lines and identify larger components making up the patterns. It should be noted, however, that local changes that reconnect stripes can have large effects on properties like the arc length and the winding number of the affected pattern components. Moreover, in a given snapshot there are only few large components. As a consequence of both aspects, these measures fluctuate more strongly than purely local measures (e.g., Ref. 16), necessitating a larger number of snapshots. In particular, in experiments this should pose no problem.

Since the approach discussed here identifies individual spirals one would expect that it would be suitable to follow the evolution of individual spirals dynamically. This would allow insight into the mechanisms that set the mean size of the spirals and may help understand the key elements that maintain the chaotic state. It has been discussed that dislocation motion and wave number selection play an important role. ${ }^{46}$ Visual inspection of the numerical results indicates that spirals shrink largely through the compression of their outer parts which then undergo a skewed-varicose instability. ${ }^{9}$

We expect that the measures introduced here can be used beneficially to investigate whether there are any transitions between different states of spiral defect chaos as $\epsilon$ (or $\operatorname{Pr}$ ) is changed. Such an investigation would require, however, a much finer scan of the parameter values, which is well beyond the reach of a computational approach. Recently, such transitions have been reported based on experimental investigations using the number of contours (Betti numbers) as the sole measure. ${ }^{32}$ We expect that our measures would provide detailed insight into the nature of these transitions. At larger $\epsilon$ a transition from spiral defect chaos to a chaotic state dominated by targets has been observed as the Prandtl number is increased beyond $\operatorname{Pr}=3.5$. $^{47,48}$ It would be interesting to see whether the additional measures introduced here shed further light on these aspects of spiral defect chaos.

In conclusion, we have introduced a new set of measures for the analysis of complex patterns, focusing on patterns that contain spiral-like structures. Our results for spiral defect chaos suggest that these measures should provide also insight into other complex states that exhibit chiral structures. Examples include meandering spirals and defect chaos in chemical systems, ${ }^{1-3,28}$ spirals in the oscillation amplitude of whirling hexagons in rotating non-Boussinesq convection, ${ }^{27}$ spirals in vertically vibrated material, ${ }^{10}$ patterns of bacterial colonies, ${ }^{6,7}$ calcium waves, ${ }^{11,12}$ spiral waves in the heart, ${ }^{49}$ and in aggregating amoebaes..$^{13,14}$

\section{ACKNOWLEDGMENTS}

We much appreciate the support by W. Pesch and his students, who developed the code we have used for the computation of the patterns. ${ }^{35}$ We also thank B. Winkler at the computing center of the University of Bayreuth for maintaining the computer system used in this work. We have benefited from discussions with G. Ahlers, G. Gunaratne, K. Krishan, K. Mischaikow, and M. Schatz. We gratefully acknowledge support from the Department of Energy (DEFG02-92ER14303) and NSF (DMS-9804673).

${ }^{1}$ M. Bär, S. Nettesheim, H. H. Rotermund, M. Eiswirth, and G. Ertl, Phys. Rev. Lett. 74, 1246 (1995).

${ }^{2}$ Q. Ouyang and J. M. Flesselles, Nature (London) 379, 143 (1996).

${ }^{3}$ A. L. Belmonte, Q. Ouyang, and J. M. Flesselles, J. Phys. II 7, 1425 (1997).

${ }^{4}$ H. Y. Guo, L. Li, Q. Y. Qi, J. Liu, and Z. S. She, J. Chem. Phys. 118, 5038 (2003).

${ }^{5}$ A. Lin, A. Hagberg, E. Meron, and H. Swinney, Phys. Rev. E 69, 066217 (2004)

${ }^{6}$ J. A. Shapiro, BioEssays 17, 597 (1995)

${ }^{7}$ H. Levine and E. Ben-Jacob, Phys. Biol. 1, 14 (2004).

${ }^{8}$ S. W. Morris, E. Bodenschatz, D. S. Cannell, and G. Ahlers, Phys. Rev. Lett. 71, 2026 (1993)

${ }^{9}$ E. Bodenschatz, W. Pesch, and G. Ahlers, Annu. Rev. Fluid Mech. 32, 709 (2000).

${ }^{10}$ J. R. Bruyn, B. C. Lewis, M. D. Shattuck, and H. L. Swinney, Phys. Rev. E 63, 041305 (2001).

${ }^{11}$ J. Lechleiter, S. Girard, E. Peralta, and D. Clapham, Science 252, 123 (1991).

${ }^{12}$ M. E. Harris-White, S. A. Zanotti, S. A. Frautschy, and A. C. Charles, J. Neurophysiol. 79, 1045 (1998).

${ }^{13}$ E. Pálsson, K. Lee, R. Goldstein, J. Franke, R. Kessin, and E. Cox, Proc. Natl. Acad. Sci. U.S.A. 94, 13719 (1997).

${ }^{14}$ D. Dormann, J.-Y. Kim, P. Devreotes, and C. Weijer, J. Cell. Sci. 114, 2513 (2001).

${ }^{15}$ R. Cakmur, D. Egolf, B. Plapp, and E. Bodenschatz, Phys. Rev. Lett. 79, 1853 (1997).

${ }^{16}$ G. H. Gunaratne, D. K. Hoffman, and D. J. Kouri, Phys. Rev. E 57, 5146 (1998).

${ }^{17} \mathrm{~S}$. Hu, G. Nathan, D. Kouri, D. Hoffman, and G. Gunaratne, Chaos 15, 043701 (2005).

${ }^{18}$ D. Egolf, I. V. Melnikov, and E. Bodenschatz, Phys. Rev. Lett. 80, 3228 (1998).

${ }^{19}$ M. Cross, D. Meiron, and Y. Tu, Chaos 4, 607 (1994).

${ }^{20}$ Y. C. Hu, R. Ecke, and G. Ahlers, Phys. Rev. E 51, 3263 (1995).

${ }^{21}$ I. Rehberg, S. Rasenat, and V. Steinberg, Phys. Rev. Lett. 62, 756 (1989).

${ }^{22}$ K. E. Daniels and E. Bodenschatz, Phys. Rev. Lett. 88, 034501 (2002).

${ }^{23}$ C. Harrison, D. H. Adamson, Z. D. Cheng, J. M. Sebastian, S. Sethura- 
man, D. A. Huse, R. A. Register, and P. M. Chaikin, Science 290, 1558 (2000).

${ }^{24}$ S. Ciliberto, P. Coullet, J. Lega, E. Pampaloni, and C. Perez-Garcia, Phys. Rev. Lett. 65, 2370 (1990).

${ }^{25}$ Y.-N. Young and H. Riecke, Phys. Rev. Lett. 90, 134502 (2003).

${ }^{26}$ L. Gil, J. Lega, and J. Meunier, Phys. Rev. A 41, 1138 (1990).

${ }^{27}$ S. Madruga, H. Riecke, and W. Pesch, Phys. Rev. Lett. 96, 074501 (2006).

${ }^{28}$ M. Hildebrand, M. Bär, and M. Eiswirth, Phys. Rev. Lett. 75, 1503 (1995).

${ }^{29}$ C. Huepe, H. Riecke, K. E. Daniels, and E. Bodenschatz, Chaos 14, 864 (2004).

${ }^{30}$ G. D. Granzow and H. Riecke, Phys. Rev. Lett. 87, 174502 (2001).

${ }^{31}$ M. Gameiro, K. Mischaikow, and W. Kalies, Phys. Rev. E 70, 035203(R) (2004).

${ }^{32}$ K. Krishan, M. Gameiro, K. Mischaikow, and M. F. Schatz (unpublished).

${ }^{33}$ F. G. Jensen, J. Sporring, M. Nielsen, and P. G. Sorensen, Chaos 12, 16 (2002).

${ }^{34}$ Y. Hu, R. Ecke, and G. Ahlers, Phys. Rev. E 55, 6928 (1997).
${ }^{35}$ W. Decker, W. Pesch, and A. Weber, Phys. Rev. Lett. 73, 648 (1994).

${ }^{36}$ W. Pesch, Chaos 6, 348 (1996).

${ }^{37}$ Y.-N. Young, H. Riecke, and W. Pesch, New J. Phys. 5, 135 (2003).

${ }^{38}$ S. Chandrasekhar, Hydrodynamic and Hydromagnetic Stability (Clarendon, Oxford, 1961).

${ }^{39}$ F. H. Busse, J. Fluid Mech. 30, 625 (1967).

${ }^{40}$ J. Liu and G. Ahlers, Phys. Rev. Lett. 77, 3126 (1996).

${ }^{41}$ R. Ecke and Y. Hu, Physica A 239, 174 (1997).

${ }^{42}$ R. E. Ecke, Y. C. Hu, R. Mainieri, and G. Ahlers, Science 269, 1704 (1995).

${ }^{43}$ G. Küppers and D. Lortz, J. Fluid Mech. 35, 609 (1969).

${ }^{44}$ Y. Hu, R. Ecke, and G. Ahlers, Phys. Rev. Lett. 74, 5040 (1995).

${ }^{45}$ S. Madruga and H. Riecke, Phys. Rev. E (submitted).

${ }^{46}$ M. C. Cross and Y. Tu, Phys. Rev. Lett. 75, 834 (1995).

${ }^{47}$ M. Assenheimer and V. Steinberg, Phys. Rev. Lett. 70, 3888 (1993).

${ }^{48}$ M. Assenheimer and V. Steinberg, Nature (London) 367, 345 (1994).

${ }^{49}$ A. X. Xu and M. R. Guevara, Chaos 8, 157 (1998). 